\title{
ChemComm
}

\section{A photoswitchable poly(3-hexylthiophene) $\dagger$}

Cite this: Chem. Commun., 2013, 49, 4637

Received 8th March 2013,

Accepted 27th March 2013

DOI: $10.1039 /$ c3cc41765d

www.rsc.org/chemcomm

A well-defined main-chain conjugated photoswitchable poly(3-hexylthiophene) with homogeneous hydrogen end groups was synthesized. Opening and closing the photoswitch enables reversible modulation of $\mathrm{P} 3 \mathrm{HT}$ emission, which is quenched by $70 \%$ when the switch is closed. The optical properties during switching cycles were quantified by irradiation/spectroscopy sequences.

Tuning the physical properties of organic semiconductors such as fluorescence, conductance energy and electron transfer by an external stimulus such as light is a rapidly growing field of research. ${ }^{1-3}$ In this respect, dithienylethene photoswitches are an important class of photochromic materials that are strongly photoresponsive and give thermally stable isomers by a reversible light driven isomerization. ${ }^{4}$ A suitable chromophore for this purpose is the 1,2-bis [5'-(phenyl)- $2^{\prime}$ methylthien-3'-yl] perfluoro-cyclopentadiene (BTF). We recently demonstrated the use of photoswitchable BTF-chromophores for potential optical transistor applications. ${ }^{5}$ Dithienylethene photoswitches coupled with molecular thiophene units can be used to trigger optical and electrochemical properties ${ }^{6,7}$ Recently, a sexithiophene with photochromic elements at both ends has been presented as a candidate for molecular wires. ${ }^{8}$

Regioregular head to tail (RR-HT) poly(3-hexylthiophene) (P3HT) is a well known hole transport material that is currently employed in various electronic devices. ${ }^{9-11}$ It has also been demonstrated that the covalent attachment of a dithienylethene photoswitch to the side chains of the P3HT copolymer resulted in amplified fluorescence quenching. ${ }^{12}$ However it is synthetically very challenging to develop well-defined conjugated polymers with very low polydispersity, and to incorporate one single photoswitchable unit into the main-chain.

\footnotetext{
${ }^{a}$ Applied Functional Polymers, Department of Macromolecular Chemistry I,

University of Bayreuth, Universitätsstrasse 30, 95440 Bayreuth, Germany.

E-mail: mukundan.thelakkat@uni-bayreuth.de; Fax: +49 (0)921 55 3109;

Tel: +49 (0921) 553108

${ }^{b}$ Lehrstuhl Experimentalphysik IV, Universitätsstraße 30, 95447 Bayreuth, Germany $\dagger$ Electronic supplementary information (ESI) available: Synthetic details including SEC, ${ }^{1} \mathrm{H}-\mathrm{NMR}$ and MALDI as well as cyclic voltammetry and thermal data. See DOI: $10.1039 / \mathrm{c} 3 \mathrm{cc} 41765 \mathrm{~d}$

† Present address: Institute of Macromolecular Chemistry, University of Freiburg, Stefan-Meier-Str. 31, 79104 Freiburg, Germany.
}

To the best of our knowledge, there is no such report on photoswitchable polymers with a single photoactive unit centered in the conjugated main chain. Owing to the capability of synthesizing mono-brominated P3HT segments via nickel-catalyzed living polymerizations, also referred to as the Grignard metathesis polymerization (GRIM) method, ${ }^{13}$ we synthesized well-defined P3HT carrying a single photoswitch moiety centered in the main chain. Such systems are highly interesting not only for switching the fluorescence behaviour of conjugated materials, but also for tuning the extent of the pi-conjugated system and hence the electronic properties.

Here, we report the synthesis and characterization of the first photoswitchable P3HT with a 1,2-bis[5'-(phenyl)-2'methylthien-3'-yl]perfluorocyclopentadiene unit (BTF) centered between two P3HT chains, via the use of the GRIM method and Suzuki coupling (Scheme 1) and the influence of switching on optical properties.

Monobrominated, narrow-distributed RR HT-P3HT 2 (GPC: $M_{\mathrm{n}}=$ $\left.1500, M_{\mathrm{w}} / M_{\mathrm{n}}=1.07\right)$ was prepared via GRIM of 2,5-dibromo-3hexylthiophene. ${ }^{14}$ Note that the synthetic methods chosen here

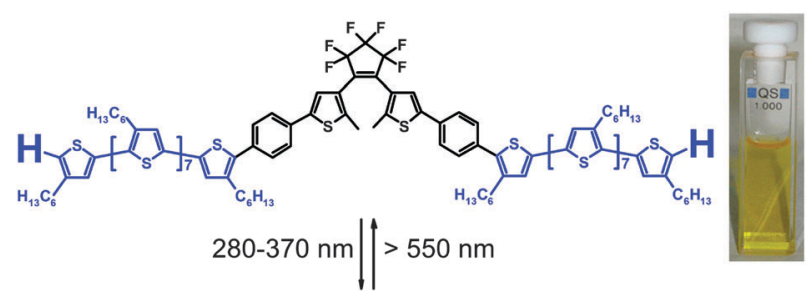

Closed form:

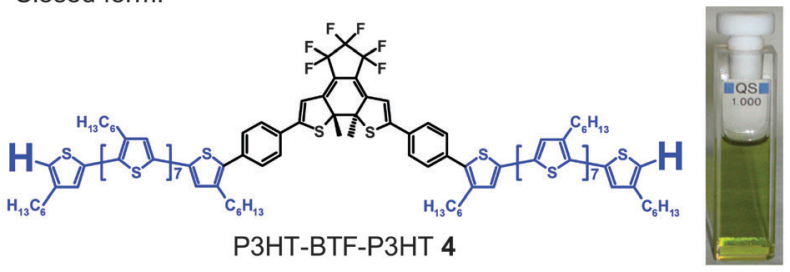

Scheme 1 Reaction scheme for the photoisomerization of the photoswitchable P3HT-BTF-P3HT 4 by photocyclization and cycloreversion of the BTF unit; ring closure occurs upon UV-irradiation and ring opening upon irradiation with visible light. The inset photos show the change in colour of solutions in toluene resulting from photocyclization of the BTF unit: open form (yellow) and closed form (green). 

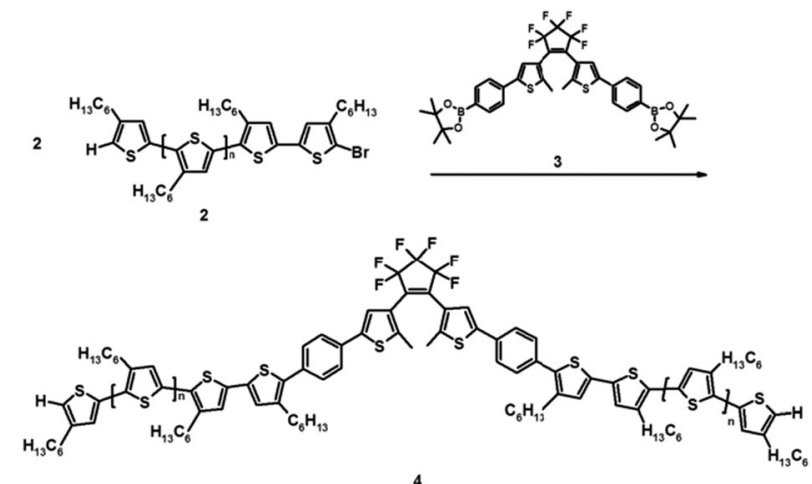

Scheme 2 Suzuki coupling of P3HT monobromide 2 and BTF bisborolane 3 to get 1,2-bis[2-methyl-5'-(4"-(poly-3-hexylthiophenyl)phenyl)thiophen-3'-yl]hexafluorocyclopenten P3HT-BTF-P3HT (4).

are not restricted to low molecular weights. The low molecular weight of 2 was intentionally chosen in order to guarantee that its absorption does not overlap with that of the BTF photoswitch in the solid state. This is important to photoaddress the moieties individually. Coupling the BTF photoswitch containing two borolane groups (3) with monobromo-P3HT 2 led to the target molecule P3HT-BTF-P3HT 4 (Scheme 2). The molecular weight determined by GPC was $M_{\mathrm{n}}=5800 \mathrm{~g} \mathrm{~mol}^{-1}$, PDI = 1.03, and MALDI-TOF showed $M_{\mathrm{n}}=3620 \mathrm{~g} \mathrm{~mol}^{-1}, \mathrm{PDI}=1.01$. It is noteworthy that the MALDI spectrum shows exclusively one peak series with homogeneous hydrogen-hydrogen endgroups (ESI $\dagger$ ). Further synthetic details are also given in the ESI. $\nmid$ Considering that we have a fully regioregular chain with a degree of polymerization of 10 and a one tail-to-tail starting unit, the regioregularity is $90 \%$. We do not expect any crystallinity in such a low molecular weight P3HT.

The optical and photochemical properties of P3HT-BTFP3HT 4 were investigated next. Scheme 1 shows the scheme of the photoisomerization of P3HT-BTF-P3HT 4 and the color in solution. The UV light induced photocyclization was initiated with a white light source $(150 \mathrm{~W})$ equipped with a Schott UG 11 band pass filter (transmittance at $334 \mathrm{~nm}>0.9$ ), whereas the cycloreversion was triggered with visible light by using a Schott OG 550 orange glass long pass filter (transmittance at $550 \mathrm{~nm}=0.5$ and at $620 \mathrm{~nm}=0.93$ ) under the same conditions. A solution of $\mathbf{4}$ in the open form is yellow, which changes to green upon irradiation with UV-light due to the ring closure of the BTF unit.

The absorption spectra recorded during the photochemical conversion (Fig. 1a) clearly show the non-structured band of the P3HT chain with $\lambda_{\max }$ at $426 \mathrm{~nm}$. This is the same as the absorption band of P3HT 2. Interestingly, there is no significant change in P3HT absorption during the photocyclization reaction. This gives a hint that ground-state absorption of the P3HT segment is not affected through the BTF unit (open as well as closed forms), which might result from the non-planarity of P3HT-BTF-P3HT 4 at the phenyl rings. In contrast, the typical additional long wave absorption band of the closed BTF unit at $\lambda_{\text {max }}=612 \mathrm{~nm}$, attributed to the extended polyene system, gains intensity with increasing UV irradiation time.

The optical properties during switching cycles of $\mathbf{4}$ were quantified by irradiation/spectroscopy sequences. For this, absorption at $612 \mathrm{~nm}$ was recorded after opening and closing the switch. a)

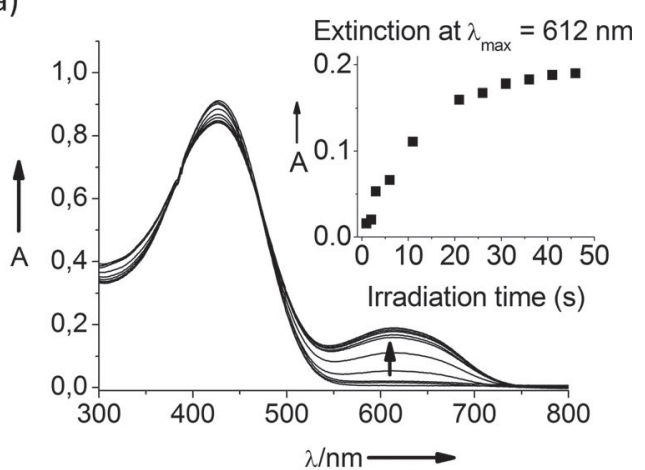

b)

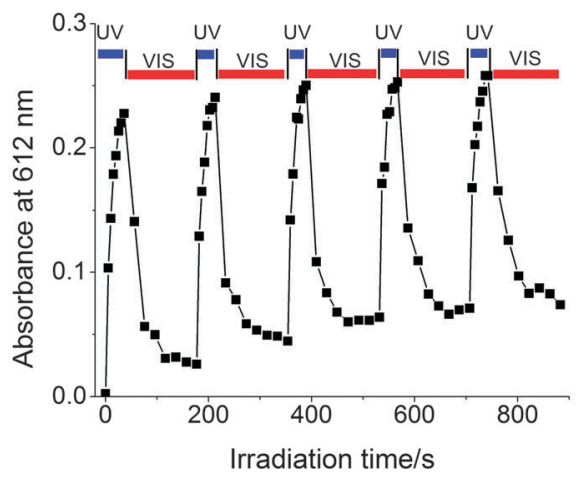

Fig. 1 (a) Normalized UV/Vis spectra of P3HT-BTF-P3HT 4 in toluene $(c=$ $0.0006 \mathrm{wt} \%)$ as a function of irradiation time. The inset shows the absorption at $612 \mathrm{~nm}$ versus irradiation time; (b) modulation of the absorbance at $612 \mathrm{~nm}$ of P3HT-BTF-P3HT 4 dissolved in toluene $\left(c=3.0 \times 10^{-6} \mathrm{wt} \%\right)$ as a function of exposure time of the UV (peak emission at $334 \mathrm{~nm}$, blue bars) and visible (peak emission at $620 \mathrm{~nm}$, red bars) irradiation.

Fig. 1b shows the change in the absorbance of P3HT-BTF-P3HT 4 probed at $612 \mathrm{~nm}$ as a function of the total exposure time of alternating UV (blue bars) and visible (red bars) irradiation. The decrease and rise of the peak absorbance at $612 \mathrm{~nm}$ demonstrates unambiguously the reversible photoisomerization of P3HT-BTFP3HT 4 and thus the switchability of the system using light.

The photoisomerization of P3HT-BTF-P3HT 4 was also investigated in films (see ESI $\dagger$ ). Here, the absorption maximum of P3HT is observed at $\lambda_{\max }=449 \mathrm{~nm}$, showing only a small bathochromic shift with respect to the UV/Vis spectra recorded in toluene $\left(\lambda_{\max }=426\right)$. This rather small change in absorption from solution to the solid state is a result of the small block length of 2, which was desired here to track the two absorption maxima of P3HT and the BTF units separately. On irradiating the sample with UV-light, the closed form of the photoswitch is generated and the corresponding absorption due to the closed BTF unit appears between 600 and $700 \mathrm{~nm}$. Annealing the film up to $200{ }^{\circ} \mathrm{C}$ neither changed the shape, nor the absorption maximum nor the intensity of absorption, indicating a complete amorphous state and the thermal stability of the system (see ESI $\dagger$ for the thermal properties investigated using DSC and TGA).

Further investigations of the switchability of the optical properties were carried out using fluorescence spectroscopy. Fig. 2 shows the fluorescence spectra of the open and the closed form in toluene. The emission peak at $555 \mathrm{~nm}$ of the open form of P3HT-BTF-P3HT 4 arises from emission of P3HT. After irradiation 


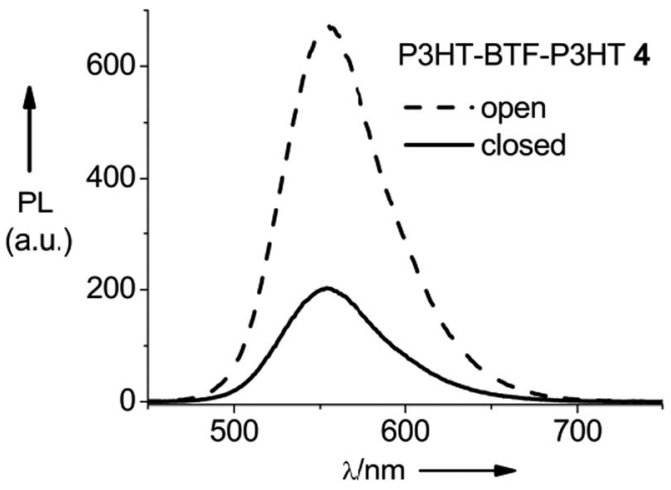

Fig. 2 Fluorescence spectra of P3HT-BTF-P3HT 4 in toluene ( $c=0.0025 w t \%)$ after excitation at $427 \mathrm{~nm}$. The closed form of 4 was obtained after irradiation for $46 \mathrm{~min}$. Emission occurs at $\lambda_{\max }=555 \mathrm{~nm}$ for both isomers, and the intensity of fluorescence is quenched by $70 \%$ in the closed form.

of 4 with UV light, the P3HT emission is quenched by $70 \%$. For comparison, the emission measurements of the photoswitch reference molecule BTF-Me 5 (BTF molecules substituted with methyl groups instead of P3HT chains, see ESI $\dagger$ ) were carried out, which show that it does not fluoresce in the open as well as in the closed form. This clearly proves that there is no contribution from the BTF unit to the fluorescence spectrum of P3HT-BTF-P3HT 4.

The basic requirement for nonradiative energy transfer from the donor P3HT to the closed BTF acceptor unit is a sufficient overlap of the emission and absorption spectra of the donor and the acceptor, respectively. Comparison of the emission spectrum of P3HT-Br 2 with the UV/Vis spectrum of the closed form of the reference molecule BTF-Me 5 reveals sufficient overlap (see ESI $\dagger$ ).

Hence, the requirements for nonradiative energy transfer according to the Förster dipole-dipole mechanism ${ }^{15}$ from P3HT to the closed BTF unit are fulfilled in P3HT-BTF-P3HT 4 (closed). Therefore, the high degree of fluorescence quenching can be explained by efficient intramolecular energy transfer from P3HT to the closed BTF switch.

We finally investigated the redox potentials of P3HT-BTFP3HT 4 and P3HT 2 by cyclic voltammetry in solution (see ESI $\dagger$ ). P3HT 2 shows a reversible oxidation onset at $0.29 \mathrm{~V} v s$. ferrocene $(\mathrm{Fc})$, similar to the first reversible oxidation of P3HT-BTF-P3HT 4 (open), which occurs at $0.24 \mathrm{~V}$ vs. Fc. The oxidation potential of the open and closed forms of the BTF unit in $\mathbf{4}$ as such could not be separately observed under the given experimental conditions. For comparison, the $\mathrm{CV}$ traces of the reference molecule BTF-Me 5 were recorded (see ESI + ). The open form shows an onset oxidation potential $E_{1}^{\mathrm{Ox}}=1.14 \mathrm{~V}$ $v s$. Fc and the closed isomer exhibits the first oxidation at $E_{1}^{\mathrm{Ox}}=$ $0.39 \mathrm{~V}$ vs. Fc, indicating that the redox potentials of the BTF bridge can be reversibly triggered by light. For P3HT-BTFP3HT 4 (open) a HOMO level of $-5.04 \mathrm{eV}$ was estimated in good agreement with the HOMO of P3HT 2 with $-5.09 \mathrm{eV}$. The LUMO level of P3HT $2(-3.32 \mathrm{eV})$ lies close to the value of P3HT-BTF-P3HT 4 (open) $(-3.44 \mathrm{eV})$. Hence, the introduction of the BTF bridge does not change the electrochemical properties of the P3HT chain in P3HT-BTF-P3HT 4 (open), which is also in accordance with the results from UV-vis spectra.

In conclusion, we have synthesised and characterised a photoswitchable P3HT-BTF-P3HT consisting of two poly(3hexylthiophene) blocks and a photoswitchable BTF unit. MALDI-TOF revealed that P3HT-BTF-P3HT is well-defined and narrow-distributed and contains uniform $\mathrm{H}-\mathrm{H}$ end groups. The photophysical properties indicate that ground-state absorption of P3HT in P3HT-BTF-P3HT 4 is not affected by the attachment of the BTF unit, while a red shift of the long wave absorption maximum of the BTF unit in the closed P3HTBTF-P3HT 4 isomer is observed. Fluorescence of the open P3HT-BTF-P3HT 4 isomer arises from P3HT emission, which is quenched by $70 \%$ in the closed $\mathrm{P} 3 \mathrm{HT}-\mathrm{BTF}-\mathrm{P} 3 \mathrm{HT}$ isomer through energy transfer after irradiating with UV light. Photoswitching in films is demonstrated as well, since the low molecular weight of the P3HT segments guarantees a small bathochromic shift of P3HT in films and therefore sufficient separation of the absorption maxima of P3HT and the closed isomer of the BTF unit. The similar HOMO levels of P3HT-BTFP3HT 4 and the closed isomer of the BTF switch are important prerequisites for switching the charge transport in thin films, making such materials interesting for device applications.

We acknowledge financial support for this work from DFG (GRK 1640).

\section{References}

1 M. Bossi, V. Belov, S. Polyakova and S. W. Hell, Angew. Chem. Int. Ed., 2006, 118, 7623.

2 A. Kronemeijer, H. B. Akkerman, T. Kudernac, B. J. van Wees, B. L. Feringa, P. W. M. Blom and B. de Boer, Adv. Mater., 2008, 20, 1467.

3 J. M. Endtner, F. Effenberger, A. Hartschuh and H. Port, J. Am. Chem. Soc., 2000, 122, 3037.

4 M. Irie, Chem. Rev., 2000, 100, 1685.

5 M. Pärs, C. C. Hofmann, K. Willinger, P. Bauer, M. Thelakkat and J. Köhler, Angew. Chem., Int. Ed., 2011, 50, 11405.

6 T. Saika, M. Irie and T. Shimidzu, J. Chem. Soc., Chem. Commun., 1994, 2123.

7 Y. Feng, Y. Yan, S. Wang, W. Zhu, S. Qian and H. Tian, J. Mater. Chem., 2006, 16, 3685.

8 J. Areephong, J. H. Hurenkamp, M. T. W. Milder, A. Meetsma, J. L. Herek, W. R. Browne and B. L. Feringa, Org. Lett., 2009, 11, 721.

9 H. Sirringhaus, P. J. Brown, R. H. Friend, M. M. Nielsen, K. Bechgaard, B. M. W. Langeveld-Voss, A. H. J. Spiering, R. A. J. Janssen, E. W. Meijer, P. Herwig and D. M. de Leeuw, Nature, 1999, 401, 685.

10 (a) G. Li, V. Shrotriya, J. Huang, Y. Yao, T. Moriarty, K. Emery and Y. Yang, Nat. Mater., 2005, 4, 864; (b) M. Sommer, S. Huettner, U. Steiner and M. Thelakkat, Appl. Phys. Lett., 2009, 95, 183308.

11 B. Li, M. C. Iovu, M. Jeffries-El, G. Sauvé, R. Zhang, J. Cooper, S. Santhanam, L. Schultz, J. C. Revelli, A. G. Kusne, T. Kowalewski, J. L. Snyder, L. E. Weiss, G. K. Fedder, R. D. McCullough and D. N. Lambeth, Nano Lett., 2006, 6, 1598.

12 J. Finden, T. K. Kunz, N. R. Branda and M. O. Wolf, Adv. Mater., 2008, 20, 1998.

13 (a) A. Yokoyama and T. Yokozawa, Chem. Rev., 2009, 109, 5595; (b) I. Osaka and R. D. McCullough, Acc. Chem. Res., 2008, 41, 1202.

14 E. E. Sheina, J. Liu, M. C. Iovu, D. W. Laird and R. D. McCullough, Macromolecules, 2004, 37, 3526.

15 M. Klessinger and J. Michl, Excited States and Photochemistry of Organic Molecules, VCH, Weinheim, 1995, p. 288. 